\title{
Experimental characterization of starting jet dynamics
}

\author{
Geno Pawlak $^{\mathrm{a}, *}$, Carolina Marugan Cruz ${ }^{\mathrm{b}}$, Carlos Martínez Bazán ${ }^{\mathrm{b}, 1}$, \\ Pedro García Hrdy ${ }^{b}$ \\ ${ }^{a}$ Department of Ocean and Resources Engineering, University of Hawai'i at Manōa, 2540 Dole Street, Holmes Hall 402, \\ Honolulu, HI 96822, USA \\ bárea de Mecánica de Fluidos, Departamento de Ingeniería Térmica y de Fluidos, Universidad Carlos III, Madrid, Spain
}

\begin{abstract}
The dynamics of a laminar starting jet are explored in a series of laboratory experiments and numerical simulations. We identify new, objective methods for characterizing the leading vortex ring, enabling robust comparisons with results from a numerical model. Observations of circulation in the initial vortex ring and for the total jet are reported along with strain rate at the leading stagnation point. Growth and pairing of shear instabilities trailing the leading vortex ring is observed. Development of these secondary vortices and their subsequent interactions with the leading vortex has significant effects on the characteristics of the primary vortex ring. Strong fluctuations in strain rate at the leading edge are associated with the pairing of the initial vortex ring with a trailing secondary ring.
\end{abstract}

Keywords: Transient jets; Starting jets; Vortex rings; Vortex pairing; Strain rate; Shear instabilities; PIV; Numerical simulations

\section{Introduction}

The initial development of a jet emanating from an orifice involves the rollup of vorticity into a vortex ring which is trailed by a column of high-momentum fluid, subject to shear instabilities. These transient or starting jets play a fundamental role in a range of engineering problems including industrial fluid flows such as fuel and oxidizer jets in combustion chambers, and pressurized vessel breaches. In addition,

\footnotetext{
* Corresponding author. Tel.: +1 8089568100 ; fax: +1 8089563498.

E-mail address: pawlak@ hawaii.edu (G. Pawlak).

${ }^{1}$ Present address: Área de Mecánica de Fluidos, Universidad de Jaén, Campus de las Lagunillas, 23071 Jaén, Spain.
} 
transient jets are prevalent in environmental settings ranging from tidal jets to animal propulsion. A large body of work has addressed the formation and evolution of the leading vortex ring, focusing particularly on the case where the duration of the jet is short relative to the development time of the ring. An extensive review on the topic has been carried out by Shariff and Leonard (1992).

More recently, a number of studies have addressed the starting or transient jet case, where the jet discharge time is comparable to or longer than the development time of the leading ring. Gharib et al. (1998) (hereafter, GRS) experimentally studied the formation of vortex rings generated with a piston/cylinder arrangement, testing a range of piston stroke to diameter ratios $(L / D)$ and velocity programs. They demonstrated the existence of a critical value for $L / D$ of approximately 4 , after which any additional circulation injected by the jet is not incorporated into the leading vortex. This critical ratio, which they referred to as the 'formation number', can also be related to the nondimensional time at which the total jet circulation equals the maximum circulation in the leading vortex ring.

Numerical studies by Rosenfeld et al. (1998) (hereafter, RRG) and Zhao et al. (2000) have examined the parameters affecting the formation number for transient jets, finding a strong dependence on the velocity profile and a weak, but significant, dependence on the velocity program. Smoothing the velocity profile can decrease the formation number, while smooth accelerations can increase the value. RRG further examined the role of Reynolds number in the range $R e=1250-2500$ and found no significant direct effects beyond the effects on the exit velocity profile. Instabilities in the trailing jet can also affect the formation of the leading ring. Zhao et al. (2000) found that trailing instabilities can accelerate detachment of the leading vortex thus reducing its final circulation and formation number.

Mixing processes in vortex rings and starting jets are of considerable interest for reactive flow applications. Shariff and Leonard (1992) relate transport and mixing in vortex rings to the two mechanisms that have been identified for mixing in shear layers: engulfment (caused by temporal/spatial growth of vortices along with fluctuations in vorticity) followed by straining and molecular diffusion. High spatial gradients, necessary for combustion, can occur in the vortex core, or at the leading stagnation point, as observed by Takagi et al. (1988), although mixing in the core region is limited by diffusional cancellation (Southerland et al., 1991). At the stagnation point, high scalar gradients coincide with high strain, which deters ignition. The evolution of the strain rate at the stagnation point associated with the leading vortex ring thus plays a key role in ignition processes for reacting flows (Choi et al., 1998) and is critical to modeling ignition of fuel-oxidizer jets.

The majority of studies thus far have focused on total and vortex circulation as well as the formation number. In this experimental and numerical study we are also interested in other features of the starting jets: the stagnation point (or vortex front), the vortex center location and the vortex pairing and vortex head engulfment by the trailing vortex. Furthermore, we will propose new methods to identify these features to enable objective comparison of experimental observations with model results.

This paper is organized as follows: in Section 2 the experimental set up is described, in Section 3 the numerical method used in the model cases is presented; analysis of the results is provided in Section 4 and Sections 5 and 6 are devoted to the discussion and conclusions, respectively.

\section{Experiments}

Experiments were conducted in a $40 \times 40 \times 100 \mathrm{~cm}$ plexiglas tank (Fig. 1) using water as the working fluid. The jet was injected horizontally through a $2 \mathrm{~cm}$ diameter orifice centered at one end of the tank and mounted flush with the wall. Flow was forced using a computer controlled piston which drove fluid 


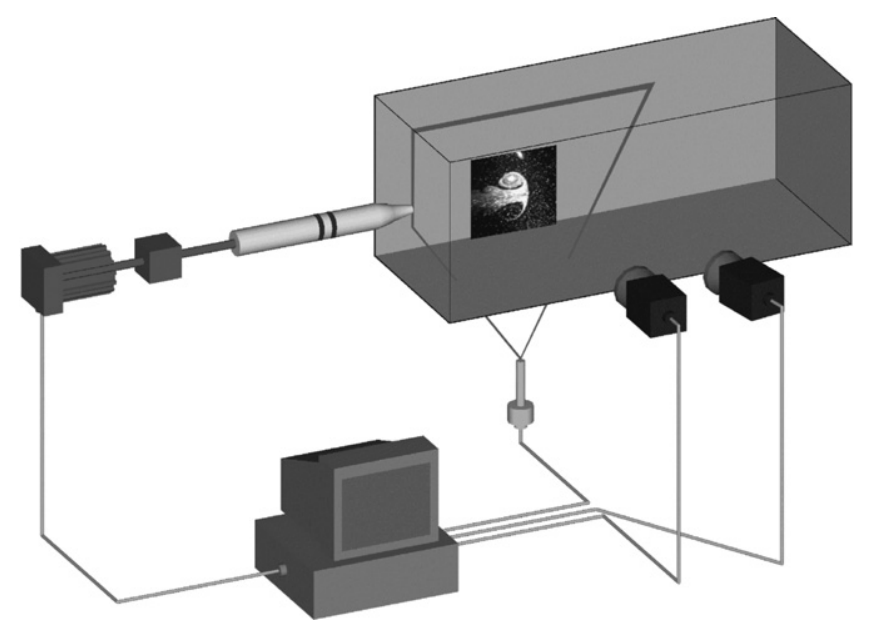

Fig. 1. Schematic of experimental facility.

through a $6.4 \mathrm{~cm}$ diameter chamber, $35 \mathrm{~cm}$ in length, into a $9.2 \mathrm{~cm}$ long converging nozzle connecting to the orifice. Care was taken in machining the components to ensure that connections between the various sections were smooth.

Water was allowed to equilibrate with the room for not less than $12 \mathrm{~h}$ prior to experiments to minimize heat transfer between the air, tank and piston, thus eliminating thermal buoyancy effects. Water in the tank was mixed thoroughly and allowed to come to rest prior to each jet realization. The top of the tank was sealed with plastic sheeting to minimize evaporative cooling at the free surface, minimizing evaporation-driven convective motions in the reservoir.

The free surface did not play a role in the dynamics of the fluid flow since the tank volume was much greater than the change in volume due to the jet influx. The speed of long waves in the tank was much greater than the jet velocities considered, ensuring that the free-surface was essentially in equilibrium on jet timescales.

The ratio of viscous timescale, which measures the time for development of a parabolic laminar profile within the entrance nozzle, to the advective timescale for the flow, is given by the jet Reynolds number, $R e=U_{\max } D / v$ where $U_{\max }$ is the maximum, steady state jet velocity and $D$ is the diameter of the orifice. For the experiments discussed here, $R e$ was of the order of $10^{3}$. The jet exit velocity for the experiments is therefore assumed to be a top hat profile at the orifice over the timescales of interests here.

Five piston velocities were considered, generating jet velocities of 4.2, 6.3, 8.3, 10.4 and $12.5 \mathrm{~cm} \mathrm{~s}^{-1}$ with jet exit Reynolds numbers of approximately 840, 1260, 1660, 2080 and 2500, respectively. Experimental parameters for nine cases are summarized in Table 1. Cases A-C were analyzed to examine the exit velocity and cases D-J were used for analysis of the jet dynamics. The piston stroke length $(8 \mathrm{~cm})$ and resulting run times $(>8 \mathrm{~s}$ ) were long enough to disregard transient effects associated with the tail-end of the jet.

The jet was illuminated from below using a narrow $(3 \mathrm{~mm})$ light sheet, generated using an $8 \mathrm{~W}$ argon-ion laser directed through a cylindrical lens, and aligned vertically along the center-line of the orifice (Fig. 1). The tank fluid was seeded using lycopodium spores (mean diameter, $28 \mu \mathrm{m}$ ), which were effectively neutrally buoyant. Images of the flow field were captured using a high speed Kodak Ektapro camera 
Table 1

Summary of experimental parameters for exit velocity (A-C) and vortex analysis (D-J) cases

\begin{tabular}{|c|c|c|c|c|c|c|}
\hline Case & $U_{\max }$ & $\operatorname{Re}_{D}$ & $x_{\text {meas }} / D$ & \multicolumn{2}{|c|}{$\Delta x / D \times \Delta y / D$} & \\
\hline A & 4.2 & 840 & 0.16 & 0.28 & & \\
\hline B & 8.3 & 1660 & 0.20 & 0.27 & & \\
\hline $\mathrm{C}$ & 10.4 & 2080 & 0.15 & 0.28 & & \\
\hline Case & $U_{\max }$ & $R e_{D}$ & $x_{0} / D$ & $x_{f} / D$ & $t_{\mathrm{v}_{0}}^{*}$ & $t_{\mathrm{v}_{f}}^{*}$ \\
\hline $\mathrm{D}$ & 6.3 & 1260 & 4.07 & 6.77 & 11.06 & 14.82 \\
\hline $\mathrm{E}$ & 8.3 & 1660 & 0.45 & 3.99 & 2.78 & 9.18 \\
\hline $\mathrm{F}$ & 8.3 & 1660 & 4.07 & 6.84 & 11.41 & 15.24 \\
\hline $\mathrm{G}$ & 10.4 & 2080 & 0.33 & 2.86 & 2.61 & 6.87 \\
\hline $\mathrm{H}$ & 10.4 & 2080 & 3.93 & 6.7 & 11.04 & 14.96 \\
\hline $\mathrm{J}$ & 12.5 & 2500 & 0.62 & 4.07 & 3.44 & 9.50 \\
\hline
\end{tabular}

which obtained 8-bit, $512 \times 480$ pixel images at 60 frames per second (fps). A second CCD camera captured $640 \times 480$ pixel, 8 -bit images at $12.5 \mathrm{fps}$ for flow visualization purposes. The high-speed camera was positioned normal to the laser sheet, with the second, slower frame rate camera positioned at a very slight angle relative to the normal direction. Image scale was determined from a reference grid imaged at the start of the experiments. The axial extent of the imaged region is given in Table 1 for cases D-J. The sixth and seventh columns list the time at which the vortex center enters, and when the stagnation point exits the imaged domain, respectively, nondimensionalized using the maximum jet velocity, $U_{\max }$ and the jet diameter, $D$. The early flow stages are captured by cases $\mathrm{E}, \mathrm{G}$ and $\mathrm{J}$ and the latter stages by cases $\mathrm{D}, \mathrm{F}$ and $\mathrm{H}$ with a small gap in between. These times will establish the boundaries for the analysis techniques described further below.

Velocity fields were obtained using a digital particle image velocimetry (DPIV) system (cf. Pawlak and Armi, 1998; Fincham and Spedding, 1997) which yields two components of velocity in a planar field. Briefly, the algorithm carries out cross-correlation analysis on two successive images of the particle field, first using a course-grid to estimate the flow velocities within $64 \times 64$ pixel interrogation windows spaced at overlapping 32 pixel intervals. The course velocity field is filtered to remove outliers, then used in a fine grid analysis that obtains estimates for the $2 \mathrm{D}$ velocities within $32 \times 32$ pixel boxes spaced at 16 pixel intervals. Sub-pixel velocity resolution is achieved using a Gaussian fit to the fine grid correlations. The resulting velocity fields are filtered using a threshold median filter to eliminate outliers. Fig. 2 shows raw and filtered vector fields for a sample case illustrating the effect of the filtering process.

The lowest level of velocity uncertainty can be estimated from the velocity scatter for regions where the velocity is expected to be zero and where gradients are weak. Using vectors obtained far from the jet axis (along the upper rows of images, for example), we find that the standard deviation of the temporal and spatial scatter in the raw velocity vectors is of the order of 0.03 pixels. This gives an estimate for minimum velocity error of the order of $0.03 \mathrm{~cm} / \mathrm{s}$ (for typical resolutions of 60 pixels $/ \mathrm{cm}$ ). The uncertainty is generally dependent of the number of particles in the sampling window, the size of the interrogation window, the particle image size and the local velocity gradients. Uncertainty further increases as particle displacements increase. Given these factors, we estimate errors for individual images as high as $10 \%$ in high shear regions of the flow. Further details on error estimates for DPIV are described by Fincham and Spedding (1997) and Willert and Gharib (1990). 


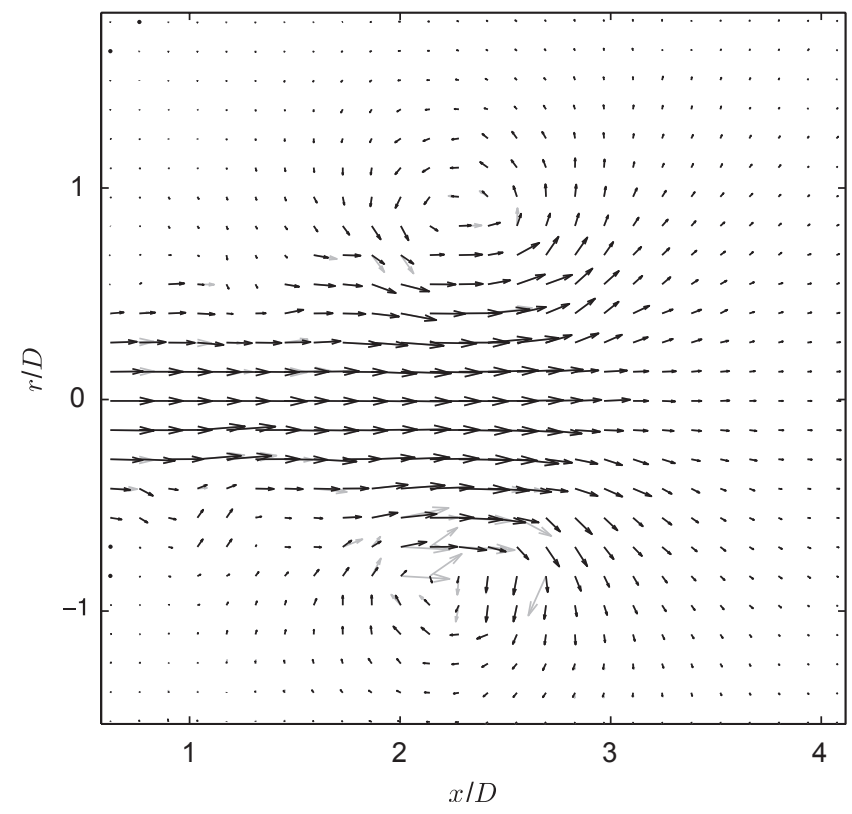

Fig. 2. Sample DPIV data. Raw velocity vectors are shown by gray arrows, with filtered velocity field shown by black arrows. Velocity field corresponds to case $\mathrm{J}, t^{*}=7.2$ (see also Fig. 5, panel 4).

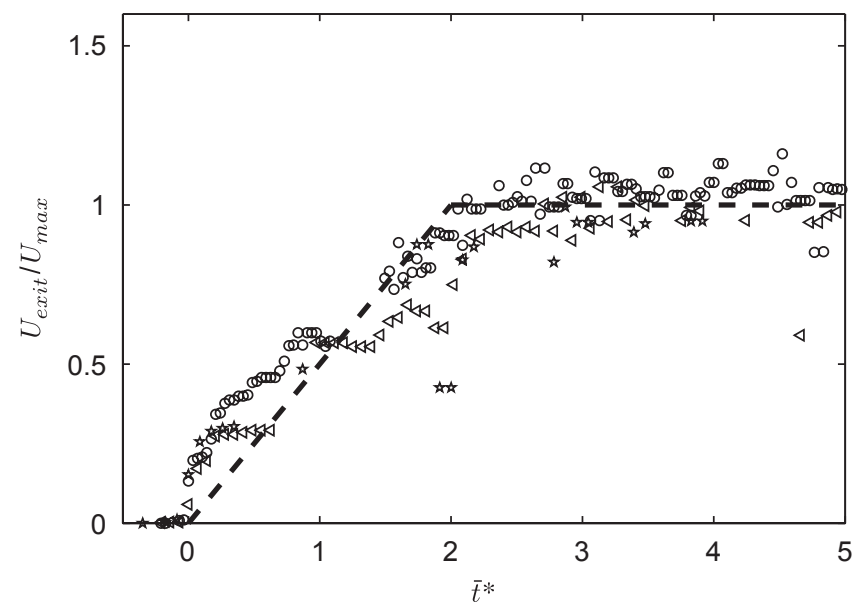

Fig. 3. Jet exit velocity versus time for experimental cases A-C. Dashed line indicates velocity evolution for low acceleration numerical model case. Symbols: 。 case A $(R e=840), \triangleleft$ case B $(R e=1660)$ and $\star$ case C $(R e=2080)$.

The nondimensional azimuthal vorticity, $\hat{\omega}_{\theta}$, can then be obtained in the laser plane from the DPIV analysis by calculating velocity derivatives:

$$
\hat{\omega}_{\theta}=\frac{D}{\bar{U}}\left(\frac{\partial u_{r}}{\partial x}-\frac{\partial u_{x}}{\partial r}\right)
$$



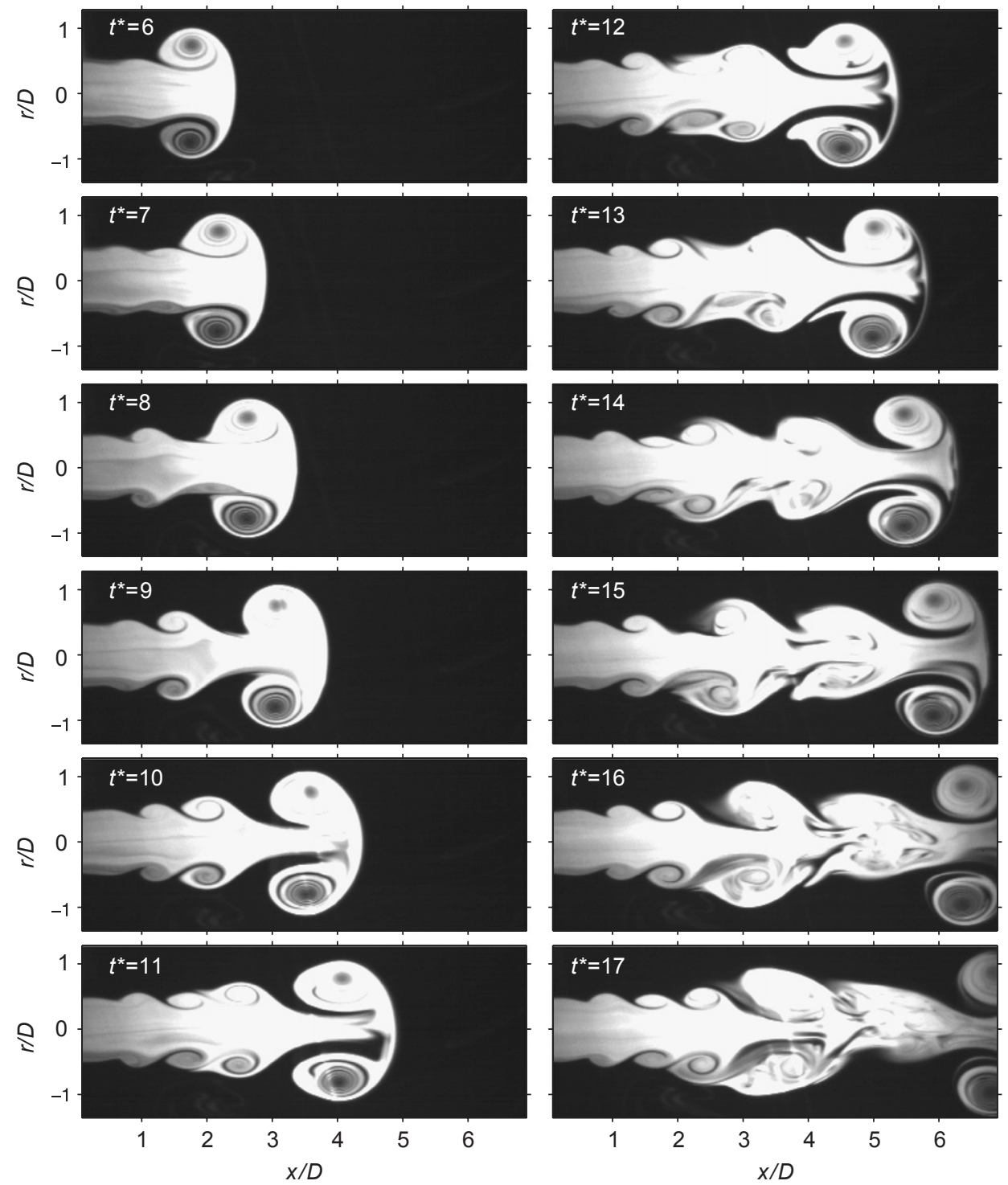

Fig. 4. Laser induced fluorescence flow visualization sequence for $R e=1660$.

where vorticity is normalized using the jet diameter, $D$, and a jet velocity scale, $\bar{U}$, defined later. The derivatives in Eq. (1) are estimated using an average of the center-differenced gradients at the desired grid point and the adjacent (lateral) points. The estimate of vorticity is equivalent, then, to calculation of the circulation at each grid point using velocities at eight surrounding points. To reduce error in vorticity measurements, velocity measurements were further median filtered in time over three successive image pairs.

The initial jet acceleration was obtained a posteriori from DPIV analysis just downstream of the jet exit for three experimental cases. Table 1 lists experimental parameters along with axial measurement 

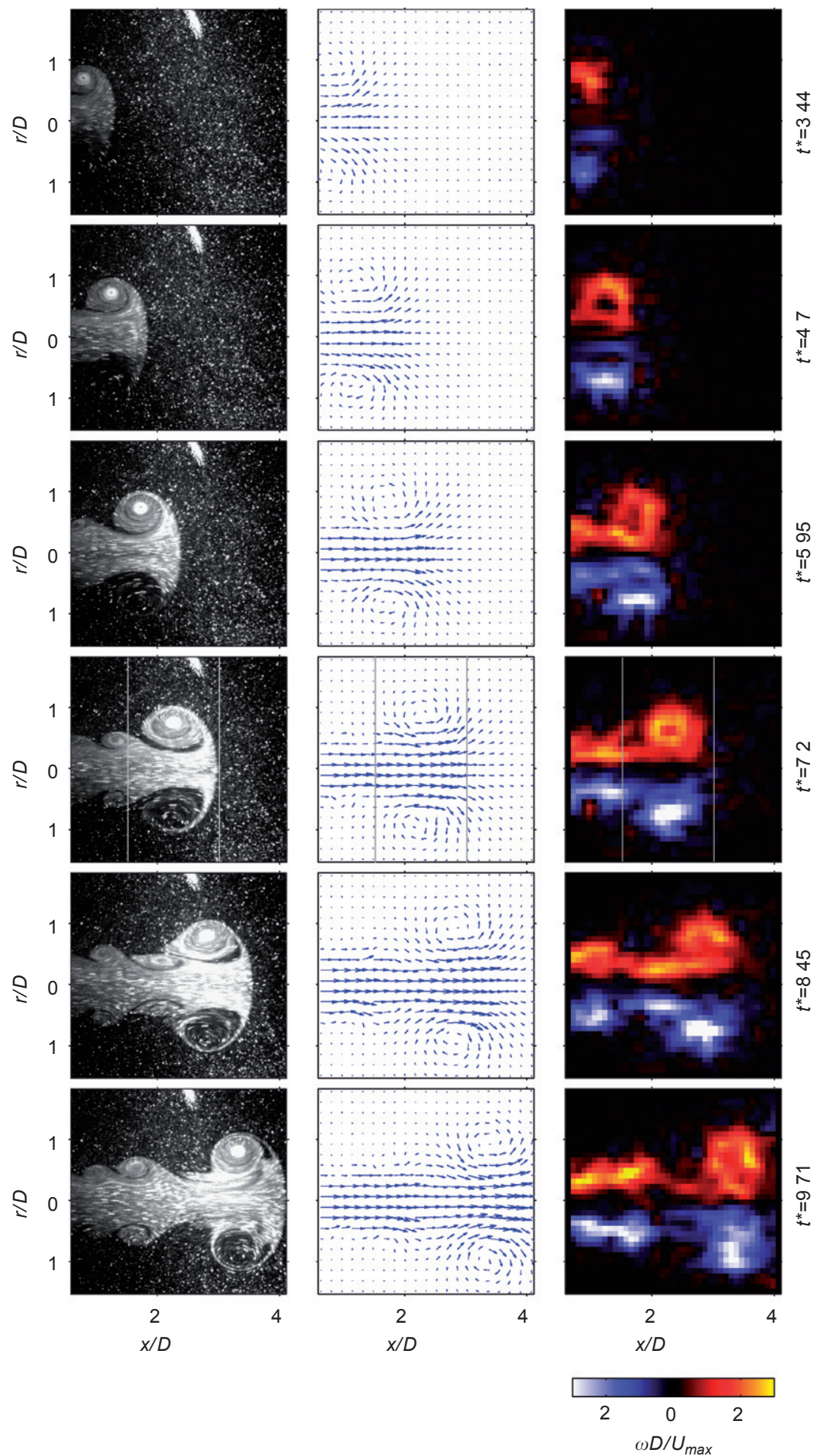

Fig. 5. Flow visualization sequence (left) with corresponding vector (center) and vorticity fields (right) for $R e=2500$ (case J). The circulation integration domain, $x_{\mathrm{v}} \pm 0.75 D$, is outlined for $t^{*}=7.2$. 
location $\left(x_{\text {meas }}\right)$ and DPIV measurement area. Fig. 3 shows the jet velocity versus time measured just downstream of the orifice for experimental cases A, B and C. These three cases adequately imaged the exit region of the jet to allow for DPIV analysis. The exit velocity data has been filtered using a three image temporal median filter to remove obvious outliers, but still appears somewhat noisy since it has not been spatially filtered as is typically done for DPIV data. Nevertheless, the moderate acceleration, reaching maximum velocity by $t^{*}=t U_{\max } / D=2$, is apparent. The acceleration profile is slightly higher for $t^{*}<1$ but the velocity is generally well-approximated by a linear ramp. Numerical model results for both moderate and high acceleration programs, discussed in more detail below, showed very similar development with the key feature being a slight delay in jet development. The differences in the two cases will be accounted for by a modified velocity scaling.

\subsection{Flow visualization}

Laser-induced fluorescence was used to visualize the evolution of the starting jet as illustrated in the sequence in Fig. 4. At $t^{*}=6$ (frame 1), the leading vortex ring is well developed, with a trailing secondary instability evident. The secondary ring subsequently pairs with the primary vortex ring, altering the structure of the leading vortex $\left(t^{*}=6-10\right)$. Two successive trailing vortex rings also pair $\left(t^{*}=9-15\right)$. In the final images of the sequence, the leading ring begins to separate from the trailing jet. At this stage in the animated sequences, it is evident that a portion of the trailing ring is entrained into the separating primary ring, although this is not as clear in the still images.

A small amount of fluorescein dye was added to the piston chamber for the DPIV cases enabling simultaneous visualization and velocity measurements of the jet. Fig. 5 shows an image sequence for $R e=2500$ for $t^{*}=3.44-9.71$ along with the corresponding velocity vectors and vorticity sequence. For clarity, the velocity vector plots show only every second vector. The sequence shows the initial formation of the leading vortex ring followed by subsequent, smaller annular shear instabilities. The second vortex is observed to pair with the leading ring (frames 5 and 6) with third and fourth instabilities pairing together following a similar evolution, as in the case shown in Fig. 4. A similar sequence was observed for all Reynolds numbers considered here. Flow visualizations at higher $R e$ than those presented here were characterized by development of secondary instabilities at smaller scales. In addition, a helical instability was observed, with pairing alternating between upper and lower vortices.

\section{Numerical model}

Experiments were compared quantitatively with numerical simulations carried out using a commercial code. The flow is simulated with a finite-volume discretization of the incompressible Navier-Stokes equations in an axisymmetric coordinate system. The equations are solved using a segregated method with a second-order implicit scheme to discretize the convective derivatives and central-differenced secondorder accurate scheme for diffusion terms. A second-order implicit temporal discretization scheme is used with a staggered control volume method to compute the face pressure.

At time $t^{*}=0$, the flow inside the computational domain is assumed to be at rest. A top hat profile is specified at the inlet for $t^{*}>0$. Two acceleration profiles were considered: an impulsive acceleration (case 1) and a moderate acceleration case (case 2). For the latter case, the jet accelerates at a constant rate, reaching full velocity at $t^{*}=2$ as shown in Fig. 3. No slip and zero velocity requirements are 

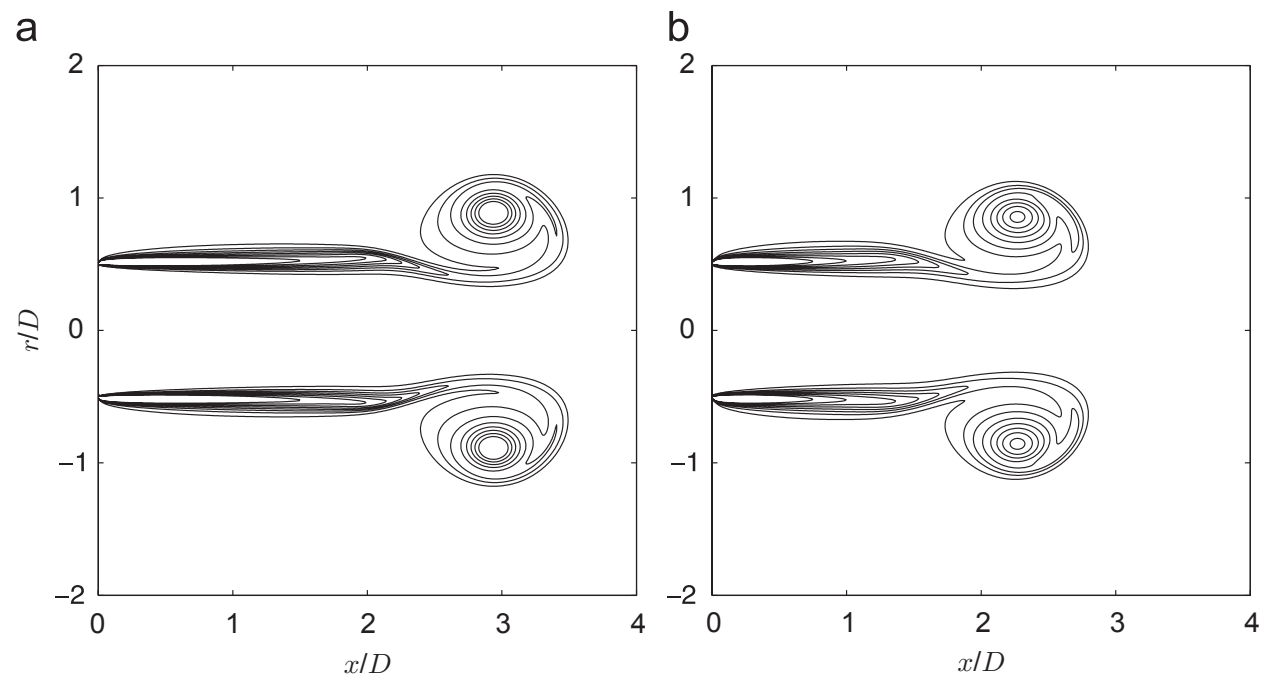

Fig. 6. Vorticity fields at $t^{*}=7.25$, for numerical model (a) case 1 and (b) case 2. Contours represent normalized vorticity, $\omega D / U_{\text {jet }}$, at intervals of 1 starting at \pm 0.5 .

imposed in the wall boundary and pressure is prescribed at the upper and front boundary. The size of the computational domain is $20 \mathrm{D}$ in the radial direction and $40 \mathrm{D}$ in the axial direction. An adaptive grid has been used to follow the jet evolution: the initial size of the grid was near 30000 cells. The adaptive criterion, based on the pressure gradient, proved to be suitable for following the jet, refining the grid at least one vortex radius ahead from the vortex front. A grid sensitivity analysis was carried out which showed that the grid size was suitable for the problem.

Vorticity fields for the two simulated cases are shown in Fig. 6. Since the model cases were unforced, secondary instabilities were notably weaker than for the experimental cases. This fact will be of use in interpreting the effects of the trailing instabilities on the evolving transient jet. The principal qualitative difference in the two cases was a delay in jet development for case 2. This is evident in the plot in Fig. 7 which shows the position of the vortex center (pressure minimum) and the stagnation point (pressure maximum) for the leading vortex ring in each case. Following GRS, we introduce a mean velocity scale defined as

$$
\bar{U}(t)=\frac{1}{t} \int_{0}^{t} U\left(t^{\prime}\right) \mathrm{d} t^{\prime}
$$

where $U(t)$ is the instantaneous jet velocity. For the acceleration profile in Fig. 3, the velocity scale is given by

$$
\bar{U}= \begin{cases}U_{\max } \frac{t^{*}}{4} & \left(0<t^{*} \leqslant 2\right), \\ U_{\max }\left(1-\frac{1}{t^{*}}\right) & \left(t^{*}>2\right),\end{cases}
$$




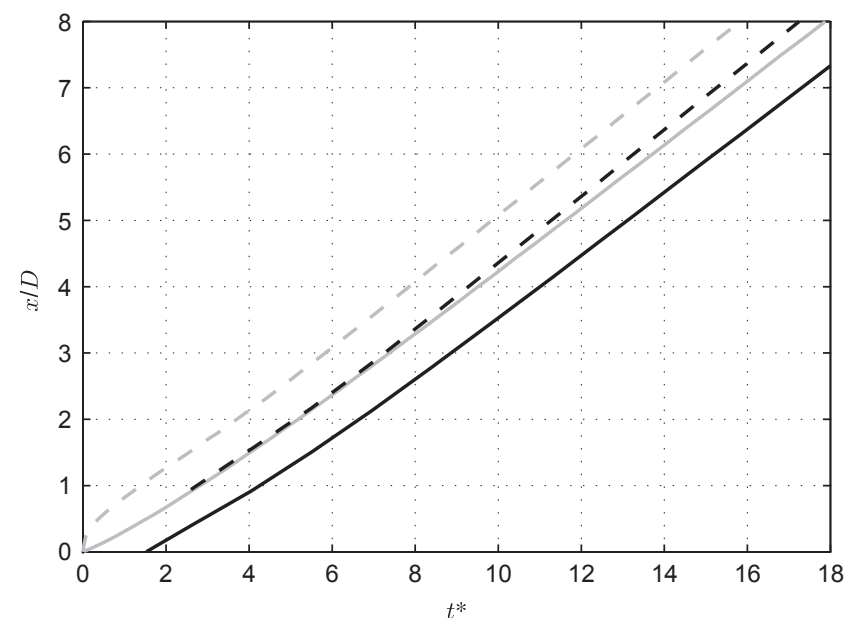

Fig. 7. Stagnation point (dashed lines) and vortex center (solid lines) position versus $t^{*}$, for model cases 1 (gray) and 2 (black).

and the resulting nondimensional time, $\bar{t}^{*}=t \bar{U} / D$, is

$$
\bar{t}^{*}= \begin{cases}\frac{t^{* 2}}{4} & \left(0<t^{*} \leqslant 2\right), \\ t^{*}\left(1-\frac{1}{t^{*}}\right) & \left(t^{*}>2\right) .\end{cases}
$$

Further variations in velocity beyond the initial ramp are neglected since, for the Reynolds number range considered, changes due to boundary layer growth in the inlet are small over the development time for the leading vortex. The nondimensional time, $\bar{t}^{*}$, referred to by GRS as the 'formation time', can also be interpreted as the length to width ratio of a cylindrical slug of fluid ejected through the orifice over time $t^{*}$. Results of analysis for the experimental results and for model case 2 will be scaled using Eqs. (3) and (4). Detailed analysis of the model results along with comparison of the experimental data is considered further below.

\section{Analysis}

A robust characterization of the leading vortex ring depends on objective and efficient methods for identifying vortex positions and length scales. Simple position estimates based on vorticity maxima or minima, for example, are subjected to errors from experimental noise in vorticity estimates. Integral approaches that exploit the inherent symmetries (and asymmetries) in the leading vortex topology can provide more robust methods for identifying vortex positions. Several such integral approaches were developed in order to minimize the effect of experimental errors and to facilitate objective comparisons with numerical simulations.

At a given instant in time, we can define a number of integral quantities for the jet that yield radially or axially varying functions and whose shapes are representative of the kinematics of the leading vortex. The physical interpretation of the functions is not of particular relevance since they are used only for objective 
identification of vortex geometry, although, in general, the integrals we examined can be identified in terms of mass and momentum fluxes.

\subsection{Axial vortex position}

To identify the axial vortex position, we examine a set of dimensionless integral functions of the axial coordinate. The first quantity given by

$$
I_{1}(x, t)=\frac{1}{\bar{U}^{2} D^{2}} \int_{0}^{R} u_{x}^{2}(r, x, t) r \mathrm{~d} r
$$

is proportional to the flux of axial momentum exiting throughout a disc of arbitrary radius $R$, perpendicular to the axial direction. The integration in Eq. (5), carried out within the imaged region and averaged over the top and bottom halves of the flow, is presented in Fig. 8(b) for a representative case (case E: $\operatorname{Re}=1660$, $\left.t^{*}=6.68\right)$. This figure shows that the axial position of the center of the leading vortex can be associated with a maximum in $I_{1}(x, t)$.

Guided by the results obtained with $I_{1}(x, t)$, we also considered the following integral functions:

$$
\begin{aligned}
& I_{2}(x, t)=\frac{1}{\bar{U}^{2} D^{2}} \int_{0}^{R} u_{r} u_{x} r \mathrm{~d} r, \\
& I_{3}(x, t)=\frac{1}{\bar{U} D^{2}} \int_{0}^{R} u_{r}(r, x, t) r \mathrm{~d} r .
\end{aligned}
$$

The first of these can be related to the flux of vertical momentum across the top or bottom half of a disc perpendicular to the axial direction. The second is responsive to the radial velocity changes around the leading vortex. Again, the physical meaning of the above quantities is not significant since we are interested in characterizing the structure of the leading vortex.

Fig. 8(b) shows the variations of these functions for the sample case in relation to the corresponding image. The shapes of the functions strongly reflect the vortex kinematics so that characteristic shapes for each quantity can be readily associated with the vortex topology. It is evident, for example, that the quantity reflected in $I_{3}$, will be positive ahead of the vortex center and negative behind, with the axial vortex position coincident with the zero crossing. Similarly, $I_{2}$ changes sign coincident with the vortex center, reflecting the outward radial flow ahead of the leading vortex. The integral in Eq. (5) reaches a maximum at the vortex center, reflecting the peak in axial momentum flux.

The three integral profiles are sensitive to experimental noise in varying degrees. In addition, transients associated with pairing also affect the correlations of the integral profiles with vortex geometry. For our experimental data, we found that $I_{3}$ was most consistent in identifying the axial vortex position. Other integral definitions can be similarly defined, although $I_{3}$ gave satisfactory results for our data sets.

Estimation of the axial position using $I_{1}$ relies on identification of the function's maximum which is, in turn, determined using the zero-crossing for $\mathrm{d} I_{1} / \mathrm{d} x$. This derivative value, proportional to $\partial u_{x} / \partial x$, results in an inherently noisier signal than $I_{2}$ and $I_{3}$ which rely instead on a direct identification of a zero-crossing. Comparing $I_{2}$ and $I_{3}$, the presence of $u_{x}$ in $I_{2}$ tends to diminish the slope near the vortex center resulting in a less robust zero-crossing estimate. $I_{3}$ thus provides the most consistent measurement due to the change in sign of $u_{r}$ at the axial location of the vortex center. 
a

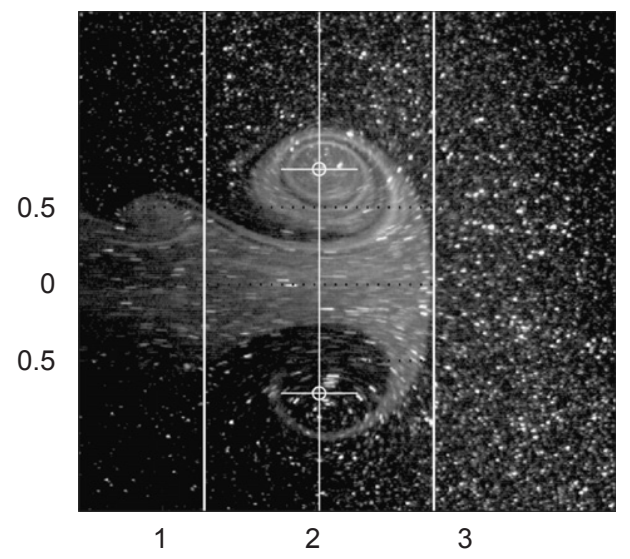

C

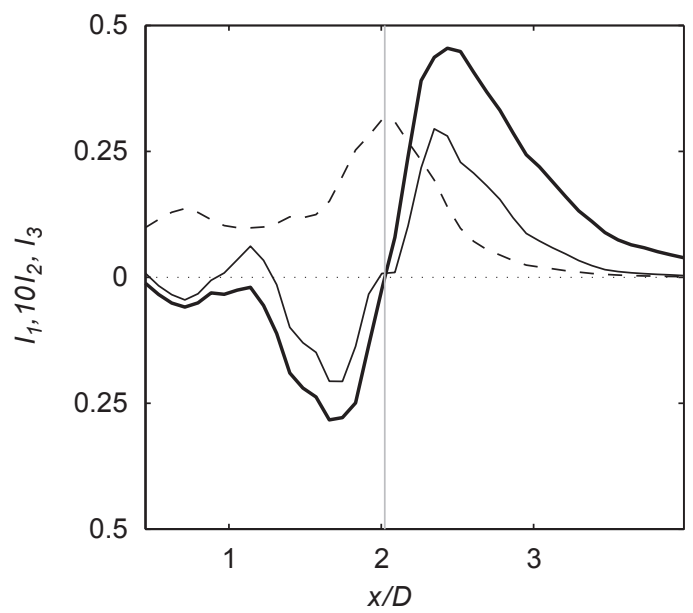

b

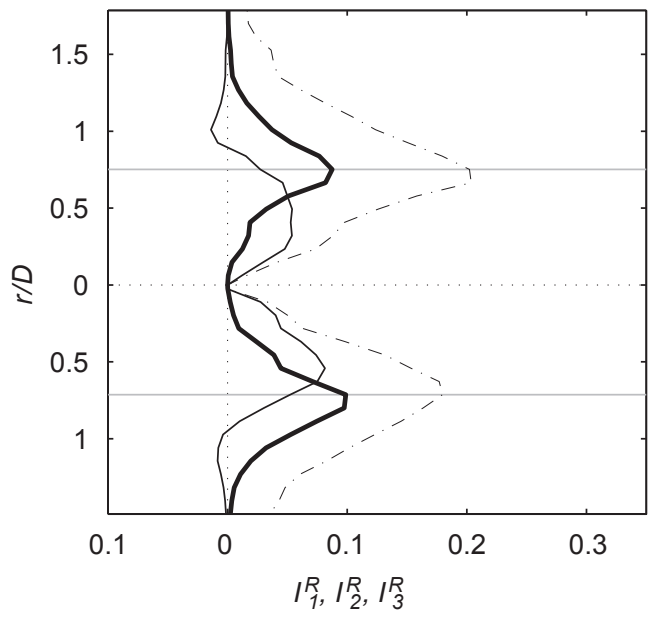

Fig. 8. (a) Image of leading vortex for $R e=1660$ (case E) at $t^{*}=6.68$. (b) Axial integral quantities versus radial position; $I_{1}$ : dashed line, $10 I_{2}$ : thin solid line, $I_{3}$ : thick solid line. (c) Radial integral quantities versus axial position; $I_{1}^{\mathrm{R}}$ : dash-dot line, $I_{2}^{\mathrm{R}}$ : thick solid line, $I_{3}^{\mathrm{R}}$ : thin solid line. Vortex center position as identified by Eqs. (7) and (9) is identified by thin horizontal lines and circles in (a) and gray lines in (b) and (c).

We define the axial vortex position, $x_{\mathrm{V}}(t)$, then, using the zero-crossing for $I_{3}$ for experimental cases D-J along with the two reference numerical model data sets. Fig. 9 shows $x_{\mathrm{v}}(t)$ calculated in this manner, with the position of minimum pressure from the numerical simulations also shown for comparison. Experimental data coverage extends from $\bar{t}^{*}=2-14$, with a short gap at $\bar{t}^{*}=9$ at the boundary between imaged regions. The axial vortex position predicted for the model cases compares very well with the location of minimum pressure, with rms errors of $0.017 \mathrm{D}$ and $0.023 \mathrm{D}$ for cases 1 and 2, respectively. For the experimental cases, the calculated vortex position was compared quantitatively with the position estimated visually (not plotted) yielding an rms error of $0.078 D$. 


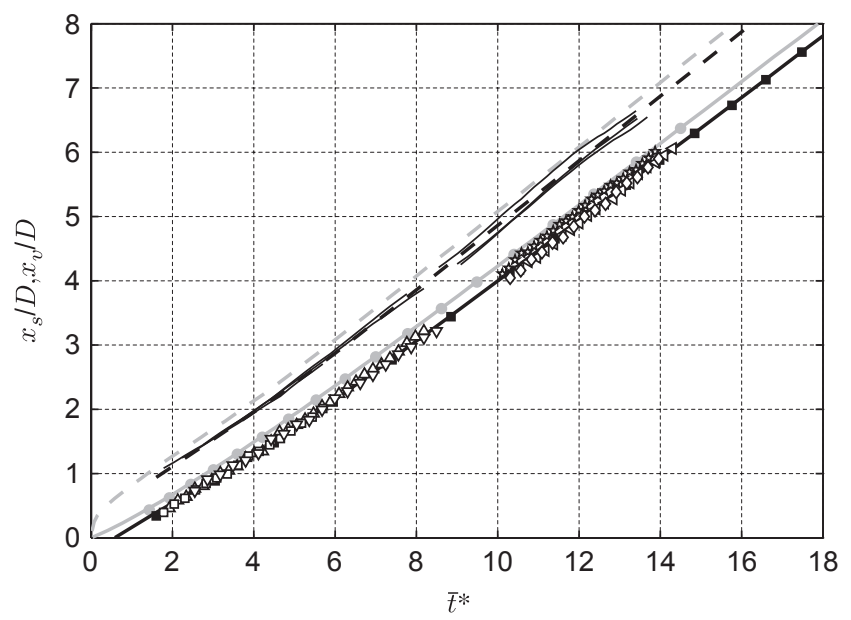

Fig. 9. Axial vortex and stagnation point position versus $\bar{t}^{*}$. Axial vortex positions estimated using Eq. (7) are represented by symbols for model and experiment cases: Model case 1: $\bullet$, model case 2: $\boldsymbol{\square}$, case D $(R e=1260): \star$ case $\mathrm{E}(R e=1660): \triangle$, case F $(R e=1660): \triangleleft$, case G $(R e=2080): \square$, case H $(R e=2090): \diamond$, case $\mathrm{J}(R e=2500): \nabla$. Pressure minimum locations are shown for model case 1 (solid gray) and case 2 (solid black). Axial stagnation point locations are shown for experiments (thin solid black), model case 1 (dashed gray) and model case 2 (dashed black). For this and subsequent plots, only every third experimental data point is shown for clarity.

We have chosen a relatively clean case in Fig. 8, for illustrative purposes. While the integral functions were not necessarily as distinctive as those shown, the objective methods provided clear identification of the vortex center in the axial direction, as illustrated by the vortex center position data in Fig. 9.

\subsection{Radial vortex position}

In a similar fashion, we can define integral quantities whose variation in the radial direction reflects the structure of the leading vortex ring. We considered the following dimensionless functions of the radial coordinate, $r$ :

$$
\begin{aligned}
& I_{1}^{\mathrm{R}}(r, t)=\frac{1}{\bar{U} D} \int_{x_{\mathrm{v}}}^{x_{\mathrm{v}}+L_{\mathrm{i}}} u_{r} \mathrm{~d} x, \\
& I_{2}^{\mathrm{R}}(r, t)=\frac{1}{\bar{U}^{2} D} \int_{x_{\mathrm{v}}-L_{\mathrm{i}}}^{x_{\mathrm{v}}+L_{\mathrm{i}}} u_{r}^{2} \mathrm{~d} x, \\
& I_{3}^{\mathrm{R}}(r, t)=\frac{1}{\bar{U}^{2} D} \int_{x_{\mathrm{v}}-L_{\mathrm{i}}}^{x_{\mathrm{v}}+L_{\mathrm{i}}} u_{r} u_{x} \mathrm{~d} x,
\end{aligned}
$$

where $L_{\mathrm{i}}$ is the axial range of integration. Here, we have chosen $L_{\mathrm{i}}=0.75 \mathrm{D}$, to encompass the leading vortex, while minimizing the influence of secondary instabilities.

The three integrals are responsive to variations in mass $(8)$ or momentum fluxes $(9,10)$ across the surface of a cylinder of radius, $r$, aligned with the $x$-axis. Fig. 8(c) illustrates the shape of each of the integral functions for the sample case considered in Section 4.1. The functions again reflect the radial 


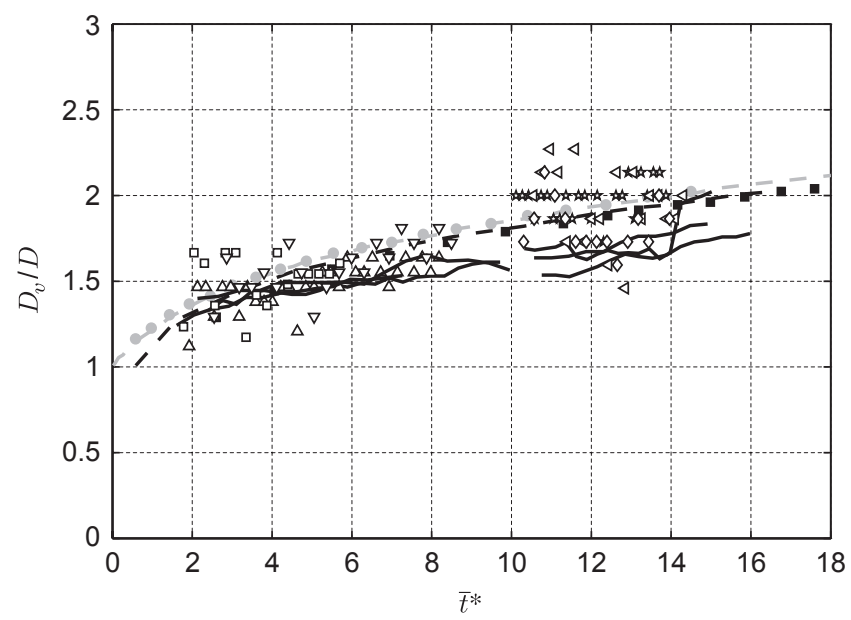

Fig. 10. Diameter of leading vortex versus $\bar{t}^{*}$. Estimates using Eq. (9) are represented by symbols for model and experiment cases. Symbols are as defined in Fig. 9. Vortex diameters defined using pressure minimum are shown for model case 1 (dashed gray) and case 2 (dashed black). Diameters estimated using visually identified vortex centers are shown by solid black lines.

structure of the leading vortex ring. Considering the kinematics of the ring, we can see that the maxima in $I_{1}^{\mathrm{R}}$ and $I_{2}^{\mathrm{R}}$ are associated with the peak radial flow coincident with the vortex center. The connection with the vortical center for $I_{3}^{\mathrm{R}}$ is less apparent since the point of zero axial velocity does not coincide with the vortex center.

For the cases considered, Eq. (9) gave the most consistent results in identifying the radial vortex position when compared with the visually identified position. We identify two vortex center radial positions with the location of the maxima in $I_{2}^{\mathrm{R}}$ in the upper and lower halves of each image. The diameter, $D_{\mathrm{v}}(t)$, of the leading vortex is then defined as the distance between these two maxima. The radial vortex position was generally clearly identified although the methods tended to overestimate the radial location relative to the visually identified center for the experimental cases ( $\mathrm{rms}$ error of $0.112 \mathrm{D}$ ). For the numerical simulations, the integrals identify the vortex position given by the pressure minimum very well (rms error of $0.006 \mathrm{D}$ and $0.009 D$ for cases 1 and 2, respectively), as shown in Fig. 10.

\subsection{Local and total circulation}

The local circulation associated with the leading vortex ring, $\Gamma_{\mathrm{L}}$, is estimated by integrating the azimuthal vorticity within a region surrounding the leading vortex defined using the objective estimates for vortex position. The integration domain is centered at the streamwise vortex position, $x_{\mathrm{v}}$, extending $\pm 0.75 \mathrm{D}$ in the streamwise direction and to the borders of the imaged region in the radial direction as illustrated in Fig. 5. The absolute value of the integrated vorticity is averaged over both halves of the image to obtain the circulation. The width of the integration band was chosen based on the vortex ring diameter scale (Fig. 10) which should also represent the streamwise length scale for the ring. Fig. 11(a) shows the evolution of $\Gamma_{\mathrm{L}}$ calculated in this manner for the experimental cases considered, along with the results for the two numerical model cases. The gap between the upstream cases (E, G and J) and the downstream cases (D, F and H) is more pronounced in this figure than in Figs. 9 and 10 due to the need to image the full integration domain. 

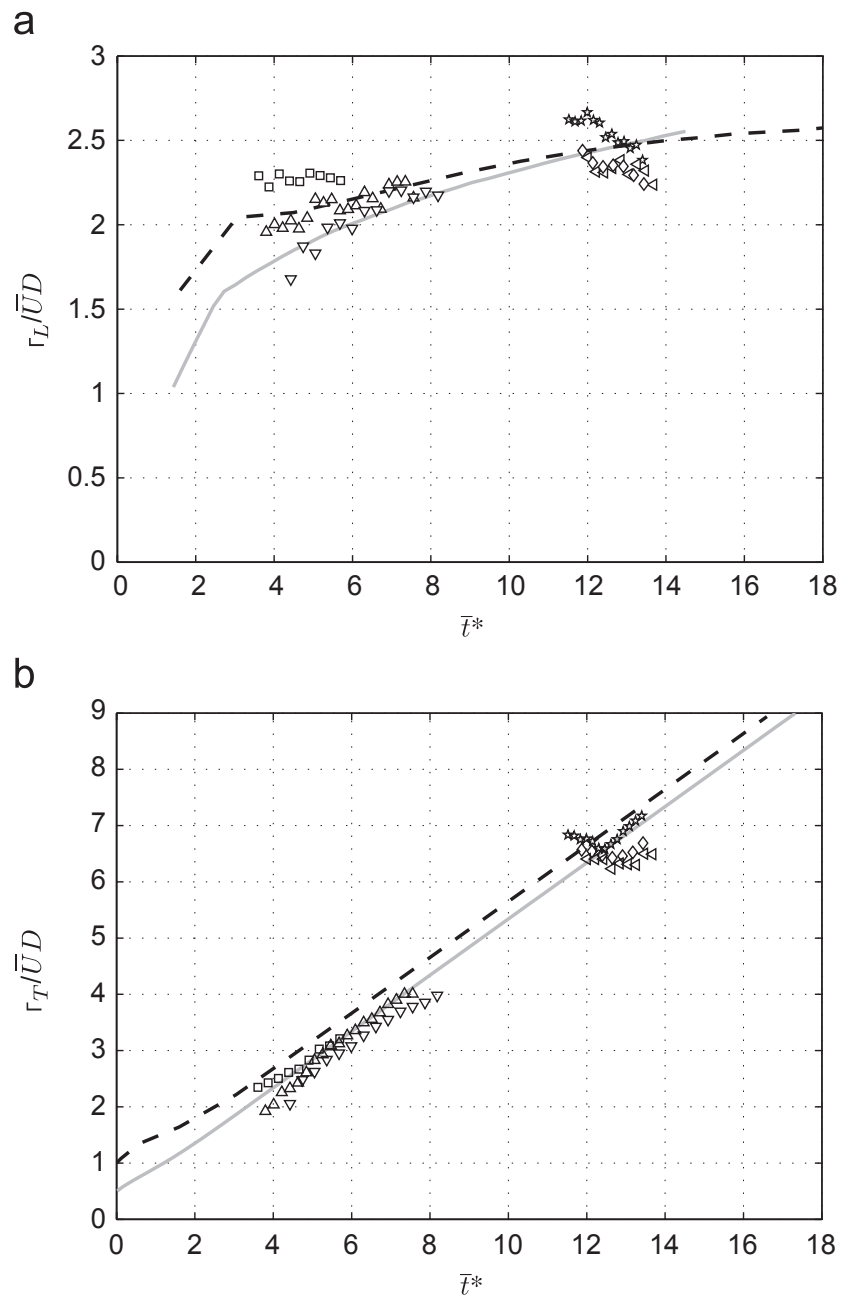

Fig. 11. (a) Local and (b) total circulation versus nondimensional time. Model cases 1 and 2 are shown by gray and black lines, respectively.

The data for the two model cases show a break in slope at about $\bar{t}^{*}=3$. This break is associated with the vortex center moving past $x / D=0.75$ after which the integration domain for $\Gamma_{\mathrm{L}}$ begins to move downstream and no longer includes the entire jet. A similar break is apparent slightly later for the experimental cases $\mathrm{E}$ and $\mathrm{J}$, although for a different reason. In these cases, after the leading vortex moves past $0.75 D$ from the left image boundary ( $\bar{t}^{*} \sim 3.7$ for case E, $\bar{t}^{*} \sim 4.1$ for case J; see Fig. 5), a secondary instability in the trailing jet begins to form outside the integration domain. The developing vortex initially accumulates some of the vorticity that would otherwise be associated with the leading vortex. As the trailing vortex forms further, it pairs with the leading vortex, increasing $\Gamma_{\mathrm{L}}$ as it enters the integration domain. The development is slightly different for case $\mathrm{G}$, where the secondary vortex development is delayed relative to the other two cases. As a result, the leading vortex accumulates additional circulation initially. A trailing vortex also pairs with the primary vortex ring, although this occurs outside the imaged 
region. Pairing processes, thus, influence the leading vortex via discrete events which have implications on the dynamics of the ring. Presumably, the timing and scale of instability development changes across the three cases as the jet Reynolds number changes.

Overall values for $\Gamma_{\mathrm{L}}$ otherwise compare quite well with model results. For the downstream cases, however, there is a discernible decay in the observed circulation, particularly for case $\mathrm{D}$. This is likely indicative of vorticity cancellation associated with asymmetries in the pairing of the trailing and leading vortex rings. From image and vorticity sequences, it appears that as the trailing vortex is amalgamated, a portion of vorticity is absorbed into the opposite side and destroyed.

The total circulation, $\Gamma_{\mathrm{T}}$, for the flow is also estimated for each experimental case. We separate the total circulation into components in and out of the imaged region. The portion, $\Gamma_{\mathrm{in}}$, within the imaged domain is given by the spatial integral of the vorticity over the imaged region, $A$,

$$
\Gamma_{\mathrm{in}}=\int_{A} \omega_{\theta} \mathrm{d} A .
$$

The portion outside the imaged region can further be separated into upstream and downstream components. Here, we will necessarily assume that the downstream contribution is negligible. The upstream contribution can be evaluated as a line integral from the jet exit, along the centerline to the start of the imaged region, vertically to the top of the tank or model domain, back to the wall at $x=0$ and down again to the jet exit. We further neglect the contributions from the latter two legs of the line integral so that the circulation outside the imaged region can be approximated as

$$
\Gamma_{\text {out }}=U_{\max } x_{\mathrm{i}}+\int_{0}^{R} u_{r}\left(x_{\mathrm{i}}, r\right) \mathrm{d} r,
$$

where $x_{\mathrm{i}}$ is the axial coordinate of the left side of the imaged domain. Here we have assumed that the velocity along the jet centerline is $U_{\max }$.

The total circulation calculated using (11) and (12) is plotted in Fig. 11(b) along with the values calculated explicitly for the model cases. The experimental values compare well, although for the far field cases, the evolution for $\Gamma_{\mathrm{T}}$ is not monotonic. This results from the discrete nature of the vorticity field along with the constant centerline velocity assumption. After the leading vortex ring enters the imaged region, there is a delay prior to the entrance of the following vortex ring, during which $\Gamma_{\text {in }}$ remains constant. As the trailing vortex ring enters the domain, $\Gamma_{\mathrm{T}}$ increases following the model values.

\subsection{Stagnation point characterization}

The stagnation point location was determined visually from the experimental image sequences. For the model cases, the stagnation point was obtained from the point of maximum pressure along the $x$-axis. Fig. 9 shows the evolution of the stagnation point location for the experimental and model cases. The experiments and model case 2 coincide well with model case 1 , using the modified timescale, $\bar{t}^{*}$, as for the axial vortex location.

An estimate of the axial strain rate at the stagnation point, $S_{\mathrm{st}}=\left(-\partial u_{x} / \partial x\right)_{x=x_{s}}$, was obtained from the experiment and model data by averaging the gradient of axial velocity over a $0.5 D \times 0.5 D$ box centered at the stagnation point location. To reduce scatter in the gradient measurement, velocities were median filtered over three successive image pairs. The $0.5 \mathrm{D}$ box size ensured a reasonable number of velocity vectors from the DPIV analysis. Using a smaller box size did not affect the magnitude for the 


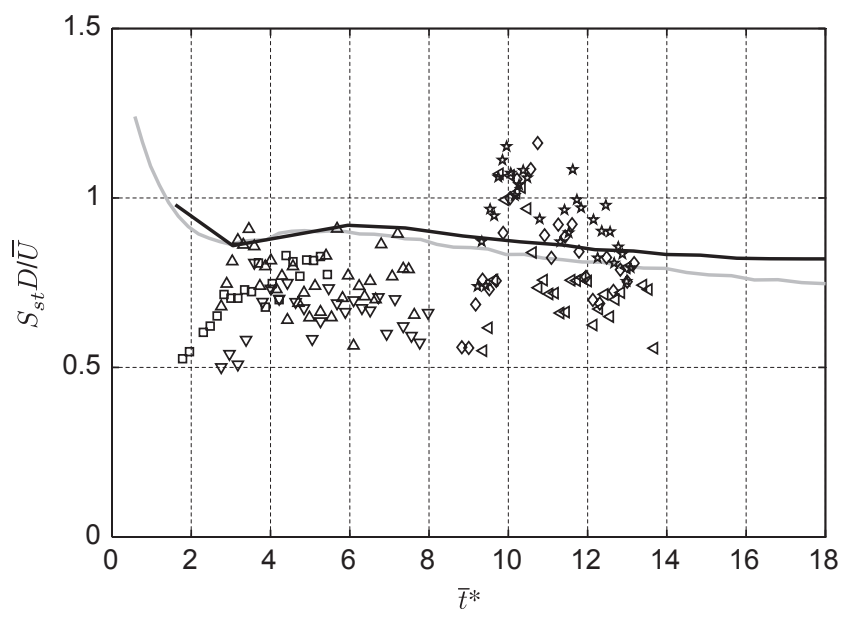

Fig. 12. Axial strain rate at the leading stagnation point for model and experimental cases. Symbols as given in Fig. 9. Model cases 1 and 2 are shown by gray and black lines, respectively.

experimental values while increasing the scatter. A larger box size did result in reduced strain measurements. This is indicative of the limitations in spatial gradient resolution for the DPIV measurements. As a result, we can expect that the strain observations somewhat underestimate the values at smaller scales.

Fig. 12 shows the evolution of $S_{\mathrm{st}}$ for the experiments and model cases. The gradient operation tends to magnify errors in the velocity estimates, so the scatter for $S_{\text {st }}$ is significant, although the model and data magnitudes compare reasonably well, with the slightly lower experimental values likely reflecting the lower spatial resolution in the experiments. Distinct features in the experimental data are maxima at $\bar{t}^{*} \approx 3-5$ and $\bar{t}^{*} \approx 10$ and the weaker values preceding these peaks. The first peak would appear to be associated with the acceleration in the exit jet velocity catching up with the leading vortex. The second, more pronounced peak can be clearly associated from the image sequence in Fig. 4 with the pairing process between the leading vortex and the trailing secondary instability. The peak in $S_{\text {st }}$ at $\bar{t}^{*}=10$ coincides with the image for $t^{*}=11$. A pronounced thinning, indicative of the high strain rate, is apparent in the image at the leading edge of the initial vortex ring, as the secondary ring vorticity is pulled within the leading vortex. The reduced strain just prior to the second peak is apparent in each of the experimental cases. This can be attributed to the weakening flow at the leading stagnation point as the initial vortex ring diameter increases just prior to pairing with the trailing secondary ring.

\section{Discussion}

The fluctuations in strain discussed in Section 4.4 have important implications on reactive flows and illustrate the importance of secondary instabilities on the flow dynamics. For the two model cases considered, lack of input forcing leads to weak secondary instabilities with little effect on the leading vortex evolution. For the experimental flows, the preferred frequencies that result from broadband forcing strongly 
affect the dynamics of the leading vortex ring with effects on the strain field at the leading stagnation point as well as on the evolution of the local circulation. It appears likely that secondary instabilities play an important role in determining the time evolution of $\Gamma_{\mathrm{L}}$, as evident by the early variations in $\Gamma_{\mathrm{L}}$ for the experimental cases discussed in Section 4.3.

For all the experimental cases considered, a single pairing event was observed within the range considered, beginning with a secondary vortex entering the $\Gamma_{\mathrm{L}}$ 'domain' $\left(x_{\mathrm{v}} \pm 0.75 D\right)$ at about $\bar{t}^{*}=5\left(t^{*}=6\right)$. The amalgamation process can be seen in Figs. 4 and 5. After the initial pairing, a second, strong, trailing vortex develops which appears to limit the supply of vorticity into the leading vortex after about $t^{*}=9$. Flow visualization of the region beyond $x / D=6$ showed some evidence of further partial pairing with the leading ring prior to pinch-off, although measurements were not carried out in this region. A part of the vorticity in the trailing vortex would then be added to the leading ring, thus increasing the circulation to values nearer to those for the model cases.

It is interesting to note that the total circulation data for the numerical cases do not intersect the vertical axis at the origin. The circulation at $t=0$ for the impulsively accelerated case has a finite value of 0.5 due to circulation generated during the initial acceleration (Iglesias et al., 2005). This is associated with the potential flow solution that applies at $t=0$, manifested initially as a vortex sheet along the vertical boundary at the inlet. Similarly, for case 2, the acceleration phase introduces additional circulation that changes the total value.

From circulation data in Figs. 11(a) and (b), we can estimate formation numbers for the various experimental and model cases considered. From the experimental data, a final value of $\Gamma_{\mathrm{L}} \approx 2.3-2.7$ would be reasonable, giving formation times, using the experimental values for $\Gamma_{\mathrm{T}}$, between roughly 3.5 and 5. Using the data for $\Gamma_{\mathrm{T}}$ for case 2, which should be more reliable given the limited extent of the DPIV measurements, formation times are between 3.2 and 4. These values are generally consistent with RRG and GRS with consideration given for the experimental error bounds. RRG also showed that nonuniform velocity profiles (due to variations in jet Reynolds number, for example) could reduce formation times. While we have not presented measurements of the exit profile here, we do not observe variations in the experimental data that are consistent with Reynolds number effects. The possible exception to this is the higher values for $\Gamma_{\mathrm{L}}$ for the low Reynolds number case D. The values of $\Gamma_{\mathrm{L}}$ are generally consistent with the values given by RRG for the uniform profile orifice case.

For the two model cases, a final value for $\Gamma_{\mathrm{L}}=2.6$ for both cases gives formation numbers of 4.5 and 3.8 for cases 1 and 2, respectively, again falling within the range cited by GRS. The lower value for case 2 is consistent with RRG's observations for nonimpulsive velocity programs. The value for case 1 lies at the upper end of the predicted range, potentially as a result of the lack of significant effects of secondary instabilities. As discussed above, these may serve to limit circulation in the leading vortex ring.

While the jet Reynolds number does not appear to influence the general results here directly, its effect on the development of secondary instabilities can be expected to be of importance. The early evolution of $\Gamma_{\mathrm{L}}$ is affected by variations in instability development (Fig. 11(a)). The timing for the pairing process within Reynolds number range considered is fairly consistent, however, as evident in the images in Figs. 4 and 5 and in the strain data in Fig. 12. Nevertheless, for a broader Reynolds number range, and potentially for jets forced preferentially at different frequencies, the scale and timing for secondary instabilities can be expected to affect the evolution of the leading vortex along with variations in strain at the leading stagnation point, with implications for reactive flows. For example, a jet might be forced to induce pairing between the trailing vortex and its trailing ring to inhibit interaction with the leading ring, thus minimizing the fluctuations in strain at the leading stagnation point. 


\section{Conclusions}

We have presented the results of experimental and numerical observations of transient jet dynamics, making use of objective integral methods for identifying vortex positions and characteristics, thus enabling robust comparisons between the lab and model results. Two numerical model cases, an impulsive case and a moderately accelerated case, were considered in order to examine the development of the idealized, unforced transient jet. An adjusted timescale defined using the time-averaged jet velocity appropriately accounts for the nonimpulsive velocity profile in the experiments. The experimental cases featured development of secondary instabilities trailing the primary vortex ring, across the range of Reynolds numbers considered, in contrast with the unforced model cases.

Integral methods for estimating axial and radial vortex positions were validated using model data. Positions estimated from the experimental data using the objective methods compared well with the model cases. Total flow circulation and local vortex circulation also compared favorably, with some deviations in the local circulation attributed to the interaction of trailing secondary vortices with the leading vortex ring. The circulation in the leading vortex increases discretely as it pairs with trailing rings. The development of these trailing instabilities and their interactions with other secondary rings thus affects the temporal evolution of the starting jet. Estimates of formation number fall within the expected range given by Gharib et al. (1998).

The evolution of the axial strain in the vicinity of the leading stagnation point was examined. Experimental values compare well with model data, although instabilities in the experiments again play an important role, introducing significant fluctuations in strain. A sharp increase in axial strain is observed as the trailing vortex pairs with the leading vortex. These variations can be expected to be important at the leading stagnation point, where high gradients compete with the high strain to determine combustive properties.

\section{Acknowledgments}

The authors are greatly indebted to Antonio Sanchez for his support and for many helpful discussions. We owe thanks as well to Javier Rodriguez for his assistance in carrying out the experiments. Support for this research was provided by the Spanish MEC and European Union under Projects \# ENE2005-08580C02-01 and DPI2005-08654-C04-01.

\section{References}

Choi, H.J., Ko, Y.S., Chung, S.H., 1998. Flame propagation along a nonpremixed vortex ring. Combust. Sci. Technol. 139, 277-292.

Fincham, A., Spedding, G., 1997. Low cost, high resolution DPIV for measurement of turbulent fluid flow. Exp. Fluids 23, 449-462.

Gharib, M., Rambod, E., Shariff, K., 1998. A universal time scale for vortex ring formation. J. Fluid Mech. 360, $121-140$. Iglesias, I., Vera, M., Sanchez, A., Liñan, A., 2005. Simulations of starting jets at low Mach numbers. Phys. Fluids 17 (3), 38105.

Pawlak, G., Armi, L., 1998. Vortex dynamics in a spatially accelerating shear layer. J. Fluid Mech. 376, 1-35.

Rosenfeld, M., Rambod, E., Gharib, M., 1998. Circulation and formation number of laminar vortex rings. J. Fluid Mech. 376, 297-318.

Shariff, K., Leonard, A., 1992. Vortex rings. Annu. Rev. Fluid Mech. 24, 235-279. 
Southerland, K.B., Porter III, J.R., Dahm, W.J.A., Buch, K.A., 1991. An experimental study of the molecular mixing process in an axisymmetric laminar vortex ring. Phys. Fluids 3 (5), 1385-1392.

Takagi, T., Okamoto, T., Ohtani, A., Komiyama, M., 1988. Measurements of velocity and concentration in transient jets by LDV and Rayleigh scattering. JSME Int. J. 31 (1), 119-126.

Willert, C.E., Gharib, M., 1990. Digital particle image velocimetry. Exp. Fluids 10 (4), 181-193.

Zhao, W., Frankel, S.H., Mongeau, L.G., 2000. Effects of trailing jet instability on vortex ring formation. Phys. Fluids 12 (3), 589-596. 